FZJ-IKP(TH)-2009-40

\title{
Lineshapes for composite particles with unstable constituents
}

\author{
C. Hanhart \\ Forschungszentrum Jülich, \\ Institut für Kernphysik (IKP-3) and Jülich Center for Hadron Physics, \\ Institute for Advanced Simulation (IAS-4), \\ D-52425 Jülich, Germany
}

Yu. S. Kalashnikova and A. V. Nefediev Institute for Theoretical and Experimental Physics, 117218, B.Cheremushkinskaya 25, Moscow, Russia

\begin{abstract}
We discuss on very general grounds possible lineshapes of composite particles with one unstable constituent. Expressions are derived in a coupled-channel formalism for constituents interacting in an $S$-wave with no assumption made on the width of one of them. We show how easy-to-use formulae, already existing in the literature, emerge for narrow constituents and identify the parameter that controls the regime of their applicability.
\end{abstract}

\section{INTRODUCTION}

In recent years charm spectroscopy had a renaissance as a result of a huge amount of data that came from modern experiments. Many new states were found at $B$-factories and Tevatron, with the bulk of them showing properties in vast conflict with what was expected from quark models of the Cornell type [1] or variants thereof - for a recent review see

Ref. [2]. The experimental situation is expected to evolve further with BES-III just having started data taking and with LHCb and PANDA on the horizon.

If there are no nearby thresholds, the lineshapes of resonances may be presented in the standard Breit-Wigner form. However, the proximity of a (relevant) threshold distorts the lineshape strongly, as was stressed recently in studies of the $X(3872)$, and then the lineshape 
of a resonance may contain important information on its nature [3, 4] - under certain conditions, discussed below, this enables one to distinguish between genuine quarkonium states and states with a significant admixture of a hadronic molecule in the wave function. However, it was found in Refs. [3, 5, 6] that the resonance lineshape might well get distorted significantly by the width of the constituents. In this paper we investigate on very general grounds how this distortion depends on the width of the constituents and under which circumstances simplified formulae for the line shapes can be used.

Indeed, the list of new near-threshold states includes, together with the above-mentioned $X$ (3872) particle, located close to the $\bar{D} D^{*}$ threshold, such entries as the $Y(4260)$ [7] (which is not far from the $D \bar{D}_{1}$ threshold, where $D_{1}$ is a $P$-wave $1^{+}$charmed meson) and the $Y(4660)$ state [8] (close to the $\psi^{\prime} f_{0}(980)$ threshold). While the width of the $D^{*}$ is tiny (less than $100 \mathrm{keV}$ ), constituent widths, generally, can be as large as $50 \mathrm{MeV}$ for $f_{0}(980)$ or even larger. In this regard, we address the following questions:

- What are the most general expressions for the lineshapes with no assumption made on the width of the constituents?

- What is the parameter that controls the deviations from the simplified expressions of Refs. [3, 5]?

The formalism employed is a straightforward generalisation of that used in Ref. [9], where only the case of stable constituents was discussed. We believe that our insights will prove useful for the analysis of future experiments.

\section{COLLECTION OF MAIN RESULTS}

In order to simplify the reading of the paper and to allow those interested in the final result only to skip all technical details, we collect in this chapter the main results and conclusions - the derivation of those is then presented in the subsequent chapters. Indeed, while the derivation of the main results turns out to be rather technical, the final conclusions are not.

For simplicity we denote the decaying particle as $X$. Although the results derived are more general and apply not only to the $X(3872)$ charmonium, we shall use the latter as a paradigmatic example for the application of the suggested approach. We use the following 
shorthand notations: in the intermediate state there are particles $a$ (stable) and $b$ (unstable, with the width $\Gamma_{R}$ ); in the final state we have the same particle $a$, while the particle $b$ decays into particles $c$ and $d$. Thus, in these notations, the process under consideration is $X \rightarrow a b \rightarrow a[c d]$ (the corresponding chain for the $X$ (3872) obviously looks like $X(3872) \rightarrow$ $\bar{D} D^{*} \rightarrow \bar{D}[D \pi]$ ). The masses of the particles are labelled accordingly (see Fig. 1). Then, as outlined below, in most relevant cases the differential rate close to the $a b$ threshold, with a relative $S$-wave between $a$ and $b$, may be written as [see Esq. (69) below]

$$
\frac{d \operatorname{Br}(a[c d])}{d E}=\mathcal{B} \frac{1}{2 \pi} \frac{g_{a b} k_{\mathrm{eff}}(E)}{\left|E-E_{X}+\frac{1}{2} g_{a b}\left[\kappa_{\mathrm{eff}}(E)+i k_{\mathrm{eff}}(E)\right]+\frac{i}{2} \Gamma_{0}\right|^{2}},
$$

where the constant $\mathcal{B}$ absorbs all details of the $X$ production, $g_{a b}$ denotes the effective coupling constant of the $X$ particle to $a b$, and $\kappa_{\text {eff }}(E)$ and $k_{\text {eff }}(E)$ parameterise the real and the imaginary part of the $X$ self-energy. The energy $E$ is counted from the three-body threshold, so that the full mass is $M=m_{a}+m_{c}+m_{d}+E$. Similarly, the parameter $E_{X}$ is related to the physical mass of the $X$ particle as $M_{X}=m_{a}+m_{c}+m_{d}+E_{X}$. In addition Eq. (11) contains $\Gamma_{0}$ to mimic the presence of extra, inelastic channels (such as $\pi^{+} \pi^{-} J / \psi$, $\pi^{+} \pi^{-} \pi^{0} J / \psi$, etc. for the $\left.X(3872)\right)$. The corresponding inelastic rate then reads [see Eq. (170) below]

$$
\frac{d \mathrm{Br}(\text { inel })}{d E}=\mathcal{B} \frac{1}{2 \pi} \frac{\Gamma_{0}}{\left.\mid E-E_{X}+\frac{1}{2} g_{a b}\left[\kappa_{\mathrm{eff}}(E)+i k_{\mathrm{eff}}(E)\right)\right]+\left.\frac{i}{2} \Gamma_{0}\right|^{2}} .
$$

For a vanishing width of the constituent, $\Gamma_{R}=0$, Eq. (1) reduces to the well-known Flattétype expression with

$$
\begin{aligned}
& k_{\text {eff }}(E)=\sqrt{2 \mu_{p}\left(E-E_{R}\right)} \Theta\left(E-E_{R}\right), \\
& \kappa_{\text {eff }}(E)=-\sqrt{2 \mu_{p}\left(E_{R}-E\right)} \Theta\left(E_{R}-E\right)+\sqrt{2 \mu_{p}\left(E_{R}-E_{X}\right)} \Theta\left(E_{R}-E_{X}\right)
\end{aligned}
$$

for the relative momentum of the $a b$ system and its analytic continuation below threshold, respectively. Here $E_{R}$ denotes the energy of the unstable constituent, also measured relative to the three-body threshold, via $m_{b}=m_{c}+m_{d}+E_{R}$, and $\mu_{p}$ denotes the reduced mass of the $a b$ system. Note that, deviating from the standard expression, $\kappa_{\text {eff }}$ was subtracted at $E=E_{X}$ in order to comply with the definition of $E_{X}$ as a zero of the real part of the inverse scattering matrix. One should keep in mind that all energies are defined with respect to the three-body threshold. However, in the limit $\Gamma_{R}=0$ this threshold becomes irrelevant and the only relevant threshold is the $a b$ two-body threshold located at $E=E_{R}$ - see the discussion below Eq. (32). 


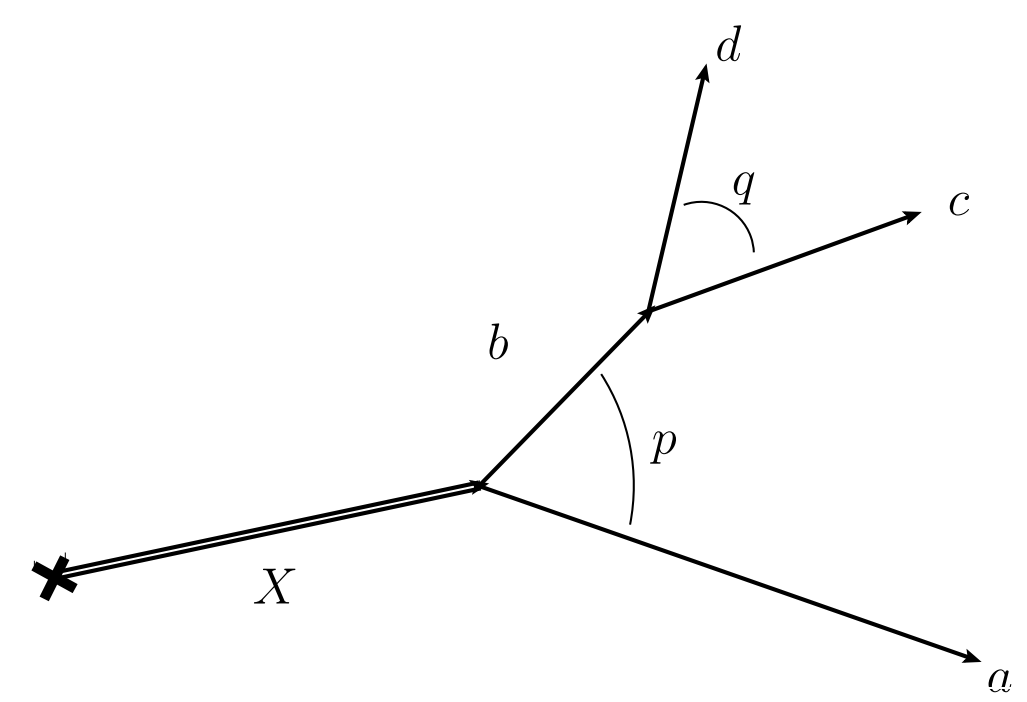

FIG. 1: Kinematics of the $X$ decay. The $X$ is assumed to be produced from a local source (denoted as a cross), and all details of its production can be absorbed into a constant $\mathcal{B}$.

Introducing a nonvanishing width $\Gamma_{R}$ implies a certain smearing of both $\kappa_{\mathrm{eff}}(E)$ and $k_{\text {eff }}(E)$, so that, in the presence of a finite width $\Gamma_{R} \neq 0$, more complicated expressions for $\kappa_{\text {eff }}(E)$ and $k_{\text {eff }}(E)$ need to be used, and we derive such expressions below [as given in Eqs. (47)-(50)]. One might expect that for an isolated, narrow constituent, distant from any threshold, the situation simplifies, and this is indeed what we observe. We therefore define the dimensionless ratio

$$
\lambda=\frac{\Gamma_{R}}{2 E_{R}}
$$

with $E_{R}$ being the energy of the unstable constituent measured with respect to the threebody threshold. In this work we only study a resonance coupling to one channel — thus the constituent is isolated by construction. The parameter $\lambda$ then captures the other two conditions, since for $\lambda \ll 1$ the width of the constituent is much smaller than the distance to the nearest threshold. It will be demonstrated below that for this situation much simpler formulae emerge - our exact expressions reduce to those presented in Ref. [5] long time ago and, more recently, in Ref. [3], namely

$$
\begin{aligned}
& k_{\mathrm{eff}}^{(0)}(E)= \sqrt{\mu_{p}} \sqrt{\sqrt{\left(E-E_{R}\right)^{2}+\Gamma^{2} / 4}+E-E_{R}} \\
& \kappa_{\mathrm{eff}}^{(0)}(E)=-\sqrt{\mu_{p}} \sqrt{\sqrt{\left(E-E_{R}\right)^{2}+\Gamma^{2} / 4}-E+E_{R}} \\
&+\sqrt{\mu_{p}} \sqrt{\sqrt{\left(E_{X}-E_{R}\right)^{2}+\Gamma^{2} / 4}-E_{X}+E_{R}},
\end{aligned}
$$


where, analogously to above, a subtraction was introduced for $\kappa_{\text {eff }}^{(0)}$. In Ref. [5] the width was taken as constant, $\Gamma \equiv \Gamma_{R}$. On the other hand, in Ref. [3] the same expressions were given, but with a "running" width,

$$
\Gamma(E)=\Gamma_{R}\left(\frac{E}{E_{R}}\right)^{l+1 / 2}
$$

where $l$ is the orbital angular momentum in the subsystem $c d$. We shall demonstrate below that for both $S$ - and $P$-wave ${ }^{1}$ resonances as constituent, in the case of $\lambda \ll 1$, Eqs. (6) and (7), when used in Eq. (11), describe the lineshapes quite accurately. In this limit the width of the resonance can safely be considered constant and thus there is no improvement to use an energy-dependent width from Ref. [3] instead. However, we shall also show that already for values as small as $\lambda \approx 0.2$ significant deviations become observable between the full expressions and the approximate ones. Especially, for the approximate expressions, and this holds for both Eqs. (6) and (77) with a constant as well as with an energy-dependent width, the amplitude shows a wrong behaviour near the three-body threshold, which is part of the signal region for values of $\lambda \approx 0.1$ and larger. The results for the lineshapes are illustrated in Figs. 6.99,

We also discuss the effect of a possible interference in the final state. For example, since the $X(3872)$ decays in equal parts via $\bar{D}^{0} D^{0 *} \rightarrow \bar{D}^{0}\left[D^{0} \pi^{0}\right]$ or $D^{0} \bar{D}^{0 *} \rightarrow D^{0}\left[\bar{D}^{0} \pi^{0}\right]$, the final states of both decay chains interfere. We argue that, to avoid a violation of unitarity, one is to include interference effects from the very beginning, by solving the full coupled-channel problem. However, for the constituent width small enough, a simple modification of Eq. (1) is possible which accounts for the interference effects, namely

$$
\frac{d \operatorname{Br}(a[c d])}{d E}=\mathcal{B} \frac{1}{2 \pi} \frac{g_{a b}\left(k_{\mathrm{eff}}(E)+k_{\mathrm{int}}(E)\right)}{\left(E-E_{X}+\frac{g_{a b}}{2} \kappa_{\mathrm{eff}}(E)\right)^{2}+\frac{1}{4}\left(\Gamma_{0}+g_{a b}\left(k_{\mathrm{eff}}(E)+k_{\mathrm{int}}(E)\right)\right)^{2}} .
$$

For a motivation for this expression see text near Eq. (79). Notice that the interference contribution $k_{\text {int }}(E)$, defined in Eq. (77), is taken into account both in the numerator and in the denominator of the differential rate as demanded by unitarity. We call this "improved" interference - to be confronted with the "naive" interference discussed in the literature,

\footnotetext{
${ }^{1}$ Notice that the $P$-wave case is relevant for the $X(3872)$, assumed to be a $D^{*} \bar{D}$ bound system, with the $D^{*}$ decaying into $\pi D$ in a $P$-wave.
} 
with the $k_{\text {int }}(E)$ term included in the numerator only [compare Eqs. (79) and (78) and see Fig. [10].

This finalises our collection of main results. Before discussing them in further detail we now first turn to their derivation.

\section{GENERALITIES}

We now start with the detailed derivation of the results presented above. For simplicity we assume that the dynamics in all subsystems can be treated nonrelativistically. Our Fock space consists of three states: a compact seed, labelled as $\left|X_{0}\right\rangle$, the $a b$ pair, and the $a[c d]$ state. Thus the wave function of the $X$ can be written as

$$
|\Psi\rangle=\left(\begin{array}{c}
C\left|X_{0}\right\rangle \\
\chi(\boldsymbol{p})|a b\rangle \\
\varphi(\boldsymbol{p}, \boldsymbol{q})|a[c d]\rangle
\end{array}\right)
$$

Here $\boldsymbol{p}$ and $\boldsymbol{q}$ are the centre-of-mass momenta in the $a b$ and $c d$ subsystems, respectively - see Fig. 1 .

Wave function (10) obeys a Schrödinger-like equation:

$$
H|\Psi\rangle=M|\Psi\rangle
$$

with the Hamiltonian

$$
H=H_{0}+V
$$

If we label the channels as

$$
1=\left|X_{0}\right\rangle, \quad 2=|a b\rangle, \quad 3=|a[c d]\rangle
$$

we may write

$$
H_{01}=M^{(0)}, \quad H_{02}=m_{a}+m_{b}^{(0)}+\frac{p^{2}}{2 \mu_{a b}}, \quad H_{03}=m_{a}+m_{c}+m_{d}+\frac{p^{2}}{2 \mu_{p}}+\frac{q^{2}}{2 \mu_{q}},
$$

with the reduced masses:

$$
\mu_{a b}=\frac{m_{a} m_{b}^{(0)}}{m_{a}+m_{b}^{(0)}}, \quad \mu_{p}=\frac{m_{a}\left(m_{c}+m_{d}\right)}{m_{a}+m_{c}+m_{d}}, \quad \mu_{q}=\frac{m_{c} m_{d}}{m_{c}+m_{d}} .
$$

The masses $M^{(0)}$ and $m_{b}^{(0)}$ entering expressions (14) and (15) are bare masses. 
Transitions allowed are $\left|X_{0}\right\rangle \leftrightarrow|a b\rangle$, mediated by the transition potential $V_{12}=V_{21}=$ $f(\boldsymbol{p})$, and $|a b\rangle \leftrightarrow|a[c d]\rangle$ (particle $a$ being a spectator), mediated by the transition potential $V_{23}=V_{32}=g(\boldsymbol{q})$. Notice that, in addition to the transition potentials $g(\boldsymbol{p})$ and $f(\boldsymbol{p})$, one might allow for a diagonal interaction in the $a b$ channel. It was shown in Refs. [10, 11], however, that nonperturbative diagonal interactions in the mesonic channels can in general be removed within effective theories employing a unitary transformation - this is not possible only in the unusual situation of a hadronic molecule and a compact state, both weakly coupled, being present near threshold simultaneously [11]. In this way all nonperturbative meson-meson interactions get cast into the effective coupling constants and the parameter $M^{(0)}$. As we shall see, the latter quantity does not play a role in the whole analysis, while the former contain important physics information - see Sec. V.

We shall focus on resonances near threshold. This allows us to use nonrelativistic kinematics and to identify $\mu_{a b}=\mu_{p}$. The latter implies that, in the expression for the reduced masses the bare mass $m_{b}^{(0)}$ is to be replaced by the physical mass $m_{b}$ (to be defined below).

Thus

$$
H=H_{0}+V=\left(\begin{array}{ccc}
M^{(0)} & f(\boldsymbol{p}) & 0 \\
f(\boldsymbol{p}) & m_{a}+m_{b}^{(0)}+T_{a b}(p) & g(\boldsymbol{q}) \\
0 & g(\boldsymbol{q}) & m_{a}+m_{c}+m_{d}+T_{a b}(p)+T_{c d}(q)
\end{array}\right),
$$

where two convenient abbreviations were introduced:

$$
T_{a b}(p)=\frac{p^{2}}{2 \mu_{p}}, \quad T_{c d}(q)=\frac{q^{2}}{2 \mu_{q}},
$$

for the kinetic energies of the $a b$ and the $c d$ subsystem, respectively.

Now one can define the fully off-shell $t$-matrix as a solution of the matrix LippmannSchwinger equation, written symbolically as

$$
t_{i k}=V_{i k}-V_{i j} \mathcal{G}_{j l}^{(0)} t_{l k}
$$

where the subscript labels channels and summation over repeated indices is understood. The free Green's function is

$$
\mathcal{G}^{(0)}=\left(H_{0}-M\right)^{-1}, \quad \mathcal{G}_{i k}^{(0)}=\delta_{i k} \mathcal{G}_{i}^{(0)}
$$

For convenience we express all energies with respect to the thresholds, thus writing

$$
M=m_{a}+m_{c}+m_{d}+E, \quad M^{(0)}=m_{a}+m_{c}+m_{d}+E_{0}, \quad m_{b}^{(0)}=m_{c}+m_{d}+E_{b}^{(0)},
$$


for the total energy $E$, the bare energy of the $X, E_{0}$, and the bare energy $E_{b}^{(0)}$ of the particle $b$, respectively.

The solution of Eq. (17) takes the form

$$
\begin{aligned}
t_{11}(E) & =\frac{G_{X}^{(0)}(E)\left(E-E_{0}\right)}{E-E_{0}+G_{X}^{(0)}(E)}, \\
t_{21}(\boldsymbol{p}, E) & =t_{12}(\boldsymbol{p}, E)=\frac{f(\boldsymbol{p}) D_{2}(p, E)}{\Delta(p, E)} \frac{E_{0}-E}{E-E_{0}+G_{X}^{(0)}(E)}, \\
t_{31}(\boldsymbol{p}, \boldsymbol{q}, E) & =t_{13}(\boldsymbol{p}, \boldsymbol{q}, E)=-\frac{g(\boldsymbol{q}) f(\boldsymbol{p})}{\Delta(p, E)} \frac{E_{0}-E}{E-E_{0}+G_{X}^{(0)}(E)}, \\
t_{22}\left(\boldsymbol{p}, \boldsymbol{p}^{\prime}, E\right) & =\delta\left(\boldsymbol{p}-\boldsymbol{p}^{\prime}\right) \frac{G_{b}^{(0)}(p, E) D_{2}(p, E)}{\Delta(p, E)} \\
& +\frac{D_{2}(p, E)}{\Delta(p, E)} \frac{f(\boldsymbol{p}) f\left(\boldsymbol{p}^{\prime}\right)}{E-E_{0}+G_{X}^{(0)}(E)} \frac{D_{2}\left(p^{\prime}, E\right)}{\Delta\left(p^{\prime}, E\right)} \\
t_{23}\left(\boldsymbol{p}, \boldsymbol{p}^{\prime}, \boldsymbol{q}^{\prime}, E\right) & =t_{32}\left(\boldsymbol{p}^{\prime}, \boldsymbol{q}^{\prime}, \boldsymbol{p}, E\right)=-\delta\left(\boldsymbol{p}-\boldsymbol{p}^{\prime}\right) \frac{g\left(\boldsymbol{q}^{\prime}\right) D_{2}\left(p^{\prime}, E\right)}{\Delta(p, E)} \\
& -\frac{g\left(\boldsymbol{q}^{\prime}\right) f(\boldsymbol{p}) f\left(\boldsymbol{p}^{\prime}\right) D_{2}(p, E)}{\Delta(p, E) \Delta\left(p^{\prime}, E\right)} \frac{1}{E-E_{0}+G_{X}^{(0)}(E)}, \\
t_{33}\left(\boldsymbol{p}, \boldsymbol{q}^{\prime}, \boldsymbol{p}^{\prime}, \boldsymbol{q}^{\prime}, E\right) & =\delta\left(\boldsymbol{p}-\boldsymbol{p}^{\prime}\right) \frac{g(\boldsymbol{q}) g\left(\boldsymbol{q}^{\prime}\right)}{\Delta(p, E)}+\frac{g(\boldsymbol{q}) g\left(\boldsymbol{q}^{\prime}\right) f(\boldsymbol{p}) f\left(\boldsymbol{p}^{\prime}\right)}{\Delta(p, E) \Delta\left(p^{\prime}, E\right)} \frac{1}{E-E_{0}+G_{X}^{(0)}(E)},
\end{aligned}
$$

where the bare self-energy parts

$$
\begin{gathered}
G_{b}^{(0)}(p, E)=\int d^{3} q \frac{g^{2}(\boldsymbol{q})}{T_{a b}(p)+T_{c d}(q)-E-i 0}, \\
G_{X}^{(0)}(E)=\int d^{3} p \frac{f^{2}(\boldsymbol{p})}{E_{b}^{(0)}+T_{a b}(p)-E-G_{b}^{(0)}(p, E)-i 0},
\end{gathered}
$$

drive the propagation of particle $b$ and the $X$, respectively, and

$$
\begin{gathered}
D_{2}(p, E)=T_{a b}(p)+E_{b}^{(0)}-E, \\
\Delta(p, E)=G_{b}^{(0)}(p, E)-D_{2}(p, E) .
\end{gathered}
$$

Let us now introduce physical masses $m_{b}$ and $M_{X}$ and the corresponding energies $E_{R}$ and $E_{X}$ as

$$
m_{b}=m_{c}+m_{d}+E_{R}, \quad M_{X}=m_{a}+m_{c}+m_{d}+E_{X} .
$$

We focus on the case of particle $b$ being a resonance, thus having $E_{R}>0$. 
The renormalised self-energies are then written as, employing subtractions at the physical masses (or energies $E_{R}$ and $E_{X}$ ),

$$
\begin{aligned}
G_{b}(p, E) & =G_{b}^{(0)}(p, E)-\operatorname{Re}\left(G_{b}^{(0)}\left(0, E_{R}\right)\right), \\
G_{X}(E) & =\int d^{3} p \frac{f^{2}(\boldsymbol{p})}{E_{R}+T_{a b}(p)-E-G_{b}(p, E)-i 0} \\
& -\operatorname{Re}\left(\int d^{3} p \frac{f^{2}(\boldsymbol{p})}{E_{R}+T_{a b}(p)-E_{X}-G_{b}\left(p, E_{X}\right)-i 0}\right) .
\end{aligned}
$$

In such a way, the physical energies $E_{R}$ and $E_{X}$ are defined as zeros of the real parts of the corresponding dressed propagators.

Note that the self-energy of the $X$ has a very peculiar analytic structure (see Ref. [12] and references therein): since $G_{b}$ has a branch point singularity at the opening of the $c d$ threshold, the $X$ self-energy shows two branch points inside the complex plane on the unphysical sheet - for the unstable resonance the physical sheet is the one where all three particles $a, c$, and $d$ are physical; the two sheets meet at the three-particle threshold. To understand the role of these singularities better, it is instructive to observe the movement of those branch points as the width of the resonance decreases: then they approach the physical axis while at the same time the coupling to the physical sheet gets weaker and weaker. In the limit of a vanishing width of the resonance, what used to be the physical sheet decouples completely converting what was the unphysical sheet to the physical sheet; at the same time the branch points merge on the physical axis to produce the branch point that starts the unitarity cut for the $a b$ channel.

Equation (11) for the wave function possesses three types of solutions (which can be defined by the corresponding asymptotic behaviour): (i) bound state of all three outgoing particles $(a, c$, and $d)$, (ii) bound state in the subsystem $c d$ and a free motion between this bound state and the particle $a$, and (iii) a free motion of all three particles, $a$, $c$, and $d$. Below we assume that neither a three-body bound state nor a bound state in the $c d$ subsystem exists, and concentrate on the solution (iii), which is the situation most relevant for the $X(3872)$ being located close to the $D^{*} D$ threshold with the $D^{*}$ as a resonance in the $D \pi$ channel.

All relevant matrix elements can be found from the multichannel $t$-matrix discussed above. However, there are no prospects for experimental studies of, say, $\bar{D} D^{*}$ elastic scattering, or of the inelastic process $\bar{D} D^{*} \rightarrow \bar{D} D \pi$. All information on the resonance $X$ is therefore 
extracted from its production from an external source. For example, from $B \rightarrow K D \bar{D} \pi$ in case of the $X(3872)$ particle. Then, if the said source can be considered as pointlike, the production amplitude may be written $\mathrm{as}^{2}$

$\mathcal{M}=\operatorname{const} \times \mathcal{G}_{1}^{(0)}(E) t_{13}(\tilde{\boldsymbol{p}}, \tilde{\boldsymbol{q}}, E)=\operatorname{const} \times \frac{f(\tilde{\boldsymbol{p}}) g(\tilde{\boldsymbol{q}})}{\left[E-E_{X}+G_{X}(E)\right]\left[E-E_{R}-T_{a b}(\tilde{p})+G_{b}(\tilde{p}, E)\right]}$,

where the details of the production dynamics are absorbed into the overall constant multiplier and the momenta $\tilde{\boldsymbol{p}}$ and $\tilde{\boldsymbol{q}}$ satisfy the total energy conservation condition,

$$
E-T_{a b}(\tilde{p})-T_{c d}(\tilde{q})=0 .
$$

For future convenience it is instructive to rederive Eq. (33) directly from the solution of Schrödinger equation (11). Indeed, for the case of a free motion of all three particles in the final state we may write

$$
\Psi_{i}=\Psi_{i}^{(0)}-\mathcal{G}_{i k}^{(0)} t_{k l} \Psi_{l}^{(0)}
$$

where $\Psi^{(0)}$ is the wave function of the free motion,

$$
\Psi_{i}^{(0)}(\boldsymbol{p}, \boldsymbol{q} \mid \tilde{\boldsymbol{p}}, \tilde{\boldsymbol{q}})=\delta_{i 3} \delta(\boldsymbol{p}-\tilde{\boldsymbol{p}}) \delta(\boldsymbol{q}-\tilde{\boldsymbol{q}}),
$$

in line with the assumed asymptotic.

The solution of Schrödinger equation (11) is

$$
\begin{aligned}
& C(\tilde{\boldsymbol{p}}, \tilde{\boldsymbol{q}})=\frac{f(\tilde{\boldsymbol{p}}) g(\tilde{\boldsymbol{q}})}{\left[E-E_{X}+G_{X}(E)\right]\left[E-E_{R}-T_{a b}(\tilde{p})+G_{b}(\tilde{p}, E)\right]}, \\
& \chi(\boldsymbol{p} \mid \tilde{\boldsymbol{p}})=\frac{C(\tilde{\boldsymbol{p}}, \tilde{\boldsymbol{q}}) f(\boldsymbol{p})+g(\tilde{\boldsymbol{q}}) \delta(\boldsymbol{p}-\tilde{\boldsymbol{p}})}{E-E_{R}-T_{a b}(p)+G_{b}(p, E)}, \\
& \varphi(\boldsymbol{p}, \boldsymbol{q} \mid \tilde{\boldsymbol{p}}, \tilde{\boldsymbol{q}})=\delta(\boldsymbol{p}-\tilde{\boldsymbol{p}}) \delta(\boldsymbol{q}-\tilde{\boldsymbol{q}})+\frac{g(\boldsymbol{q}) \chi(\boldsymbol{p} \mid \tilde{\boldsymbol{p}})}{E-T_{a b}(p)-T_{c d}(q)} .
\end{aligned}
$$

Notice that the wave functions are expressed in terms of physical energies $E_{X}$ and $E_{R}$.

\footnotetext{
${ }^{2}$ More correctly this is the expression for the production through the quark-antiquark component of the $X$ wave function (see the discussion in Ref. [13]) or if there is only a hadronic molecule present. It might be distorted significantly, if a weakly coupled molecular state and a quark state are present simultaneously in the near-threshold region - see Ref. [11]. However, since this is a quite exotic scenario we do not consider this case further here.
} 
It turns out then that, in practical applications, the quantity of an immediate relevance is the so-called spectral density, which can be built as

$$
W(E)=\int d^{3} \tilde{p} d^{3} \tilde{q}|C(\tilde{\boldsymbol{p}}, \tilde{\boldsymbol{q}})|^{2} \delta\left(E-T_{a b}(\tilde{p})-T_{c d}(\tilde{q})\right)
$$

and which measures the probability to find the bare $\left|X_{0}\right\rangle$ state in the $|a[c d]\rangle$ continuum see Sec. V] An explicit calculation gives

$$
W(E)=\frac{1}{2 \pi i}\left[\frac{1}{E-E_{X}+G_{X}^{*}(E)}-\frac{1}{E-E_{X}+G_{X}(E)}\right] .
$$

Comparing Eqs. (33) and (37) one can find for the differential rate

$$
\frac{d \operatorname{Br}(a[c d])}{d E} \propto \int d^{3} \tilde{p} d^{3} \tilde{q}|C(\tilde{\boldsymbol{p}}, \tilde{\boldsymbol{q}})|^{2} \delta\left(E-T_{a b}(\tilde{p})-T_{c d}(\tilde{q})\right)=W(E) .
$$

\section{SELF-ENERGY CALCULATION}

For energies $E$ small enough one can neglect the form factors - then the resulting shapes show universal, model-independent patterns. In this limit we may write

$$
\begin{aligned}
& G_{b}(p, E)=\frac{i}{2} g_{c d}\left[2 \mu_{q}\left(E-\frac{p^{2}}{2 \mu_{p}}\right)\right]^{l+1 / 2} \Theta\left(E-\frac{p^{2}}{2 \mu_{p}}\right) \\
& +\frac{1}{2}(-1)^{l} g_{c d}\left[-2 \mu_{q}\left(E-\frac{p^{2}}{2 \mu_{p}}\right)\right]^{l+1 / 2} \Theta\left(-E+\frac{p^{2}}{2 \mu_{p}}\right),
\end{aligned}
$$

where $l$ is orbital angular momentum in the $c d$ subsystem, and the coupling $g_{c d}$ is related to the physical resonance width $\Gamma_{R}$ via

$$
\Gamma_{R}=g_{c d}\left(2 \mu_{q} E_{R}\right)^{l+1 / 2}=g_{l} E_{R}^{l+1 / 2},
$$

where, in order to simplify the notations, the shorthand $g_{l}=g_{c d}\left(2 \mu_{q}\right)^{l+1 / 2}$ was introduced. Notice that, once we always stay in the vicinity of the resonance, we retain in Eq. (43) only the part which changes appreciably in the region of interest, while a nearly constant (infinite) contribution is omitted in Eq. (43) as it can always be got rid of through an appropriate redefinition of the physical quantity $E_{R}$. We should also stress that the case of $l \neq 0$ is more subtle, as the form factor $f(q)$ is proportional to $q^{l}$, so that the renormalisation procedure requires more than one subtraction. However, as mentioned here we are primarily interested in the leading nonanalytical near-threshold contribution from the $[c d]$ self-energy loop, which is given by expression (43). 
Thus, the two parameters we treat as input are the energy $E_{R}$ and the width $\Gamma_{R}$. An important dimensionless parameter which governs the dynamics is

$$
\lambda=\frac{\Gamma_{R}}{2 E_{R}} .
$$

We assume $\lambda<1$ - only then one can speak of the resonance as a constituent ${ }^{3}$ — and shall be primarily interested in the case $\lambda \ll 1$.

We stick to zero orbital angular momentum in the $a b$ subsystem. It is then convenient to express $G_{X}(E)$ in terms of the coupling $g_{a b}$ as

$$
\operatorname{Re}\left(G_{X}(E)\right)=\frac{1}{2} g_{a b} \kappa_{\mathrm{eff}}(E), \quad \operatorname{Im}\left(G_{X}(E)\right)=\frac{1}{2} g_{a b} k_{\mathrm{eff}}(E), \quad g_{a b}=8 \pi^{2} \mu_{p} f^{2}(0) .
$$

For a resonance in the $c d$ subsystem one finds by an explicit evaluation of $G_{X}(E)$, as given in Eq. (32), for the imaginary part

$$
k_{\mathrm{eff}}(E)=\frac{g_{l}}{2 \pi \mu_{p}} \int_{0}^{\sqrt{2 \mu_{p} E}} \frac{p^{2} d p\left(E-\frac{p^{2}}{2 \mu_{p}}\right)^{(2 l+1) / 2}}{\left(E_{R}-E+\frac{p^{2}}{2 \mu_{p}}\right)^{2}+\frac{g_{l}^{2}}{4}\left(E-\frac{p^{2}}{2 \mu_{p}}\right)^{2 l+1}},
$$

where $l$ denotes the angular momentum of the $c d$ system in its rest frame and $g_{l}$ was defined in Eq. (44). The real part is

$$
\kappa_{\text {eff }}(E)=\kappa_{1}(E)+\kappa_{2}(E)-\kappa_{1}\left(E_{X}\right)-\kappa_{2}\left(E_{X}\right),
$$

where the first term takes the form

$$
\kappa_{1}(E)=\frac{1}{\pi \mu_{p}} \int_{0}^{\infty} p^{2} d p \frac{E_{R}-E+\frac{p^{2}}{2 \mu_{p}}}{\left(E_{R}-E+\frac{p^{2}}{2 \mu_{p}}\right)^{2}+\frac{g_{l}^{2}}{4}\left(E-\frac{p^{2}}{2 \mu}\right)^{2 l+1}},
$$

and the second term is

$$
\kappa_{2}(E)=-\frac{g_{l}}{2 \pi \mu_{p}} \int_{\sqrt{2 \mu_{p} E}}^{\infty} p^{2} d p \frac{\left(\frac{p^{2}}{2 \mu_{p}}-E\right)^{(2 l+1) / 2}}{\left(E_{R}-E+\frac{p^{2}}{2 \mu_{p}}\right)^{2}+\frac{g_{l}^{2}}{4}\left(E-\frac{p^{2}}{2 \mu}\right)^{2 l+1}} .
$$

Notice that $\kappa_{2}(E)$ is suppressed as compared to $\kappa_{1}(E)$ by a factor $g_{l} \propto \lambda$, which is small by assumption. We neglect $\kappa_{2}(E)$ in what follows throughout the paper.

For example, for $l=0$ a straightforward evaluation gives

$$
k_{\mathrm{eff}}(E)=\frac{g_{0} \sqrt{2 \mu_{p}}}{2}\left(-1+\frac{E_{R}+\sqrt{\left(E-E_{R}\right)^{2}+\frac{g_{0}^{2} E}{4}}}{\sqrt{2 E_{R}\left(E_{R}-E+\frac{g_{0}^{2} E}{8 E_{R}}+\sqrt{\left(E-E_{R}\right)^{2}+\frac{g_{0}^{2} E}{4}}\right)}}\right)
$$

\footnotetext{
${ }^{3}$ For a discussion of the scattering amplitude for general values of $\lambda$ see Ref. [14].
} 

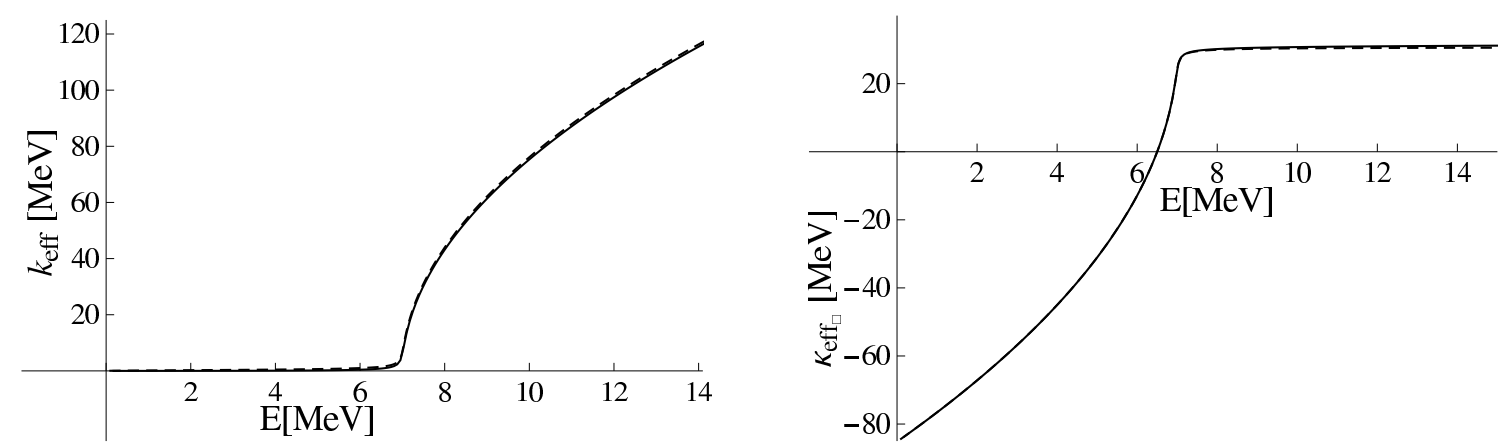

FIG. 2: The exact results for $k_{\mathrm{eff}}(E)$ and $\kappa_{\mathrm{eff}}(E)$ for the case $l=0$ versus the simplified formulae (6) and (7) with an energy dependent width as proposed in Ref. [3] for $E_{R}=7 \mathrm{MeV}$ and $\Gamma_{R}=0.1 \mathrm{MeV}$ $(\lambda=1 / 140)$. The left plot is for $k_{\text {eff }}(E)$, the right plot is for $\kappa_{\text {eff }}(E)$. The $\kappa_{2}$ term is neglected.

and

$$
\kappa_{\mathrm{eff}}(E) \approx \kappa_{1}(E)-\kappa_{1}\left(E_{X}\right)
$$

with

$$
\kappa_{1}(E)=\text { const }+\sqrt{\mu_{p}} \frac{E-E_{R}+\frac{g_{0}^{2}}{4}-\sqrt{\left(E_{R}-E\right)^{2}+\frac{g_{0}^{2} E}{4}}}{\sqrt{E_{R}-E-\frac{g_{0}^{2}}{8}+\sqrt{\left(E_{R}-E\right)^{2}+\frac{g_{0}^{2} E}{4}}}} .
$$

Notice that the (infinite) constant is cancelled in $\kappa_{\text {eff }}$, as follows from Eq. (52).

For $l>0$ the integrand in $\kappa_{\text {eff }}$ shows, in addition to the two physical poles, spurious poles in the region $p \gg \sqrt{2 \mu_{p} E}$. Their appearance is an artifact of the formalism used [in particular, a consequence of neglecting the form factor in expression (31) for the selfenergy of particle $b]$. However, since these poles are well outside the region of validity of the formalism discussed, in what follows we neglect their contribution (using the principal value prescription). Then analytical expressions for the $k_{\text {eff }}$ and $\kappa_{\text {eff }}$ can be found, in analogy with the case of $l=0$. However, such expressions are bulky, so we do not give them here and resort to numerical calculations instead.

To demonstrate quantitatively the comparison of Eqs. (47), (48) versus Eqs. (66), (77) for the sake of concreteness in all plots we use the energy-dependent width as proposed in Ref. [3] - we choose two possible parameter sets. As the first example we choose the parameters as they apply to the case of the $X(3872)$ : for the width of particle $b$ we take an empirical value of order of the $D^{*}$ state width, $\Gamma_{R}=0.1 \mathrm{MeV}$. In addition, the mass of the $D^{* 0}$ is located at about $E_{R}=7 \mathrm{MeV}$ above the $D^{0} \pi^{0}$ threshold. This corresponds 

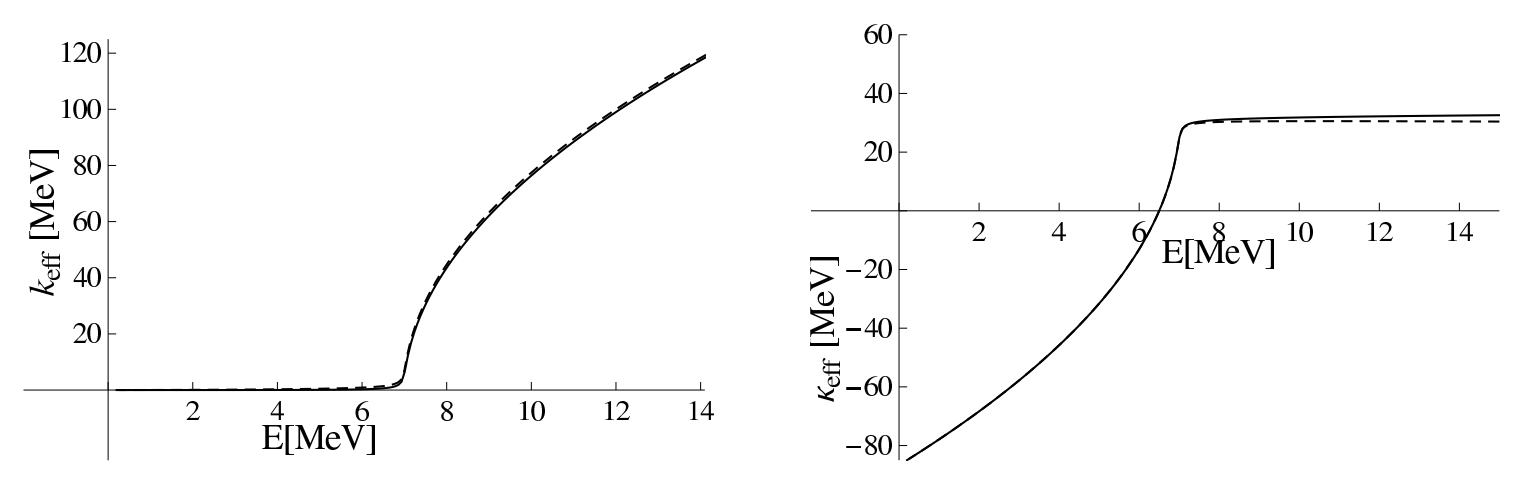

FIG. 3: The same as in Fig. 2, but for $l=1$.
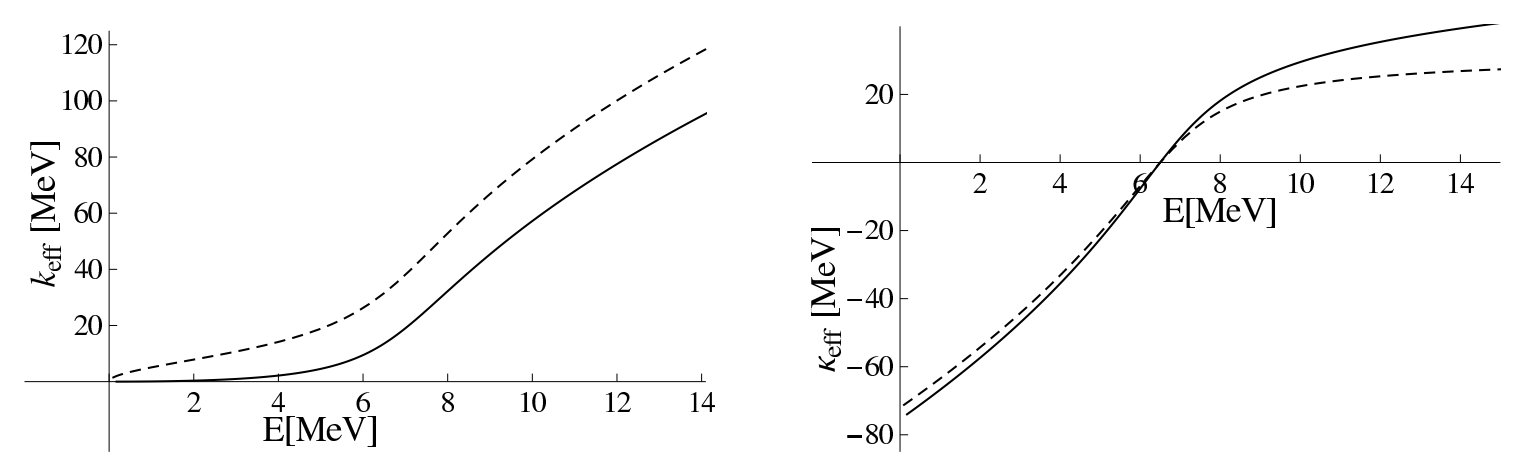

FIG. 4: The same as in Fig 2, but for $\Gamma_{R}=3 \mathrm{MeV}(\lambda=0.2)$.

to $\lambda=1 / 140$. The resulting energy dependencies of both $k_{\text {eff }}(E)$ and $\kappa_{\text {eff }}(E)$ are given in Figs. 2 and 3 for $S$ - $(l=0)$ and $P$-waves $(l=1)$, respectively. Note that the latter case is relevant for the $X(3872)$, with the $D^{*}$ decaying into $D \pi$ in a $P$-wave. Since we subtracted both expressions for $\kappa_{\text {eff }}(E)$ at the same point $E=E_{X}$, the two curves agree exactly at the subtraction point, which is chosen here as $E_{X}=E_{R}-0.5 \mathrm{MeV}$. Clearly, for the parameters relevant for the $X(3872)$ the results agree perfectly well. However, already for a width of only about $1 \mathrm{MeV}$, deviations between the formulae start to become visible. In Figs. 4 and 5 we compare the two formalisms for $\Gamma_{R}=3 \mathrm{MeV}$ ( or $\lambda \simeq 1 / 5$ ) with all other parameters unchanged. Now the difference, especially in $k_{\text {eff }}$, is sizable, mainly as a result of the very different threshold behaviour. Indeed, at the three-body threshold, Eq. (47) behaves as

$$
k_{\mathrm{eff}}(E) \underset{E \rightarrow 0}{\approx} \frac{1}{2} \Gamma_{R} \sqrt{\frac{\mu_{p}}{2 E_{R}}}\left(\frac{E}{2 E_{R}}\right)^{l+2} \propto E^{l+2},
$$

in line with the three-body phase space and the centrifugal barrier.

In the meantime, $k_{\text {eff }}^{(0)}(E)$, as given by Eq. (6) , shows a wrong threshold behaviour. If a 

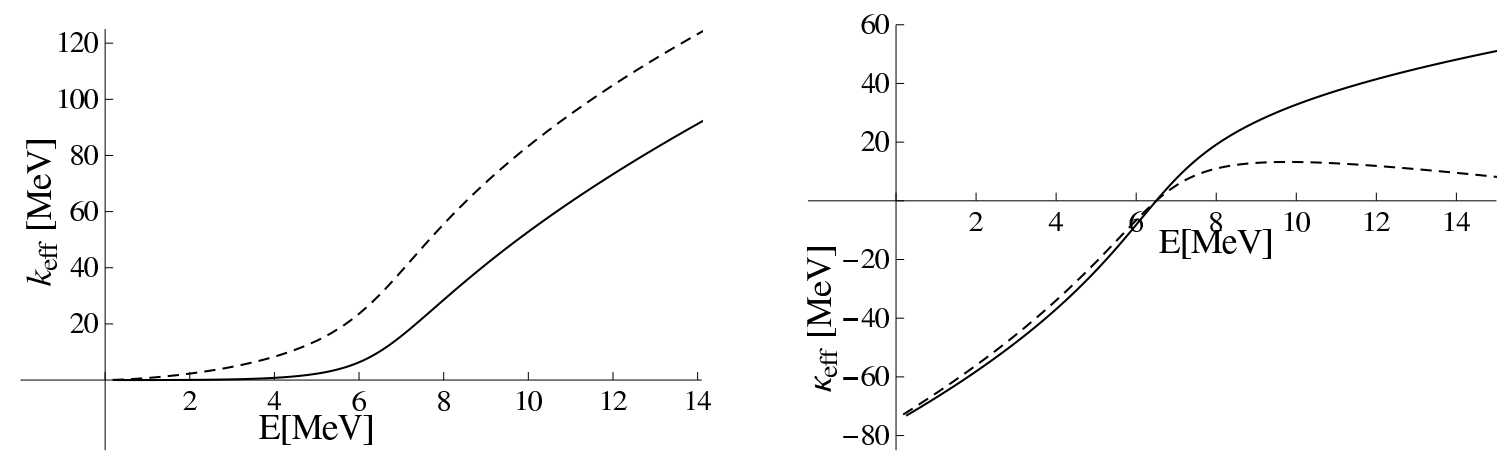

FIG. 5: The same as in Fig. 4, but for $l=1$.

constant width is used for the resonance (this case is not shown in the figures), there remains a finite imaginary part even at the threshold, while for the energy-dependent width (8) of Ref. [3] one finds

$$
k_{\text {eff }}^{(0)}(E) \underset{E \rightarrow 0}{\approx} \frac{\Gamma_{R} \sqrt{2 \mu_{p} E}}{4 E_{R}}\left(\frac{E}{E_{R}}\right)^{l} \propto E^{(2 l+1) / 2} .
$$

Thus, instead of the required behaviour according to the three-body phase space, the approximate result scales as the two-body phase space.

One concludes therefore that, if the resonance is well separated from the three-body threshold, the simple formulae (66) and (7) suggested in Ref. [5] may be used instead of Eqs. (47) and (48). Indeed, it is easy to verify that, in the limit

$$
\lambda \ll 1, \quad \frac{\left|E_{R}-E\right|}{E_{R}} \ll 1,
$$

our formulae reduce naturally to Eqs. (6), (17) [it is an easy task to check this reduction analytically for the $S$-wave case - see Eqs. (51) and (52)]. Notice that in this case the width of particle $b$ can safely be taken as constant in Eqs. (6) and (7) and there is no improvement to use an energy-dependent width from Ref. [3] instead. This is definitely so for the parameters relevant for the $X(3872)$. However, when investigating particles with broader constituents, one has to resort to the full formulae (47) and (49). This applies, in particular, in studies of the $Y(4260)$. The $Y(4260)$ resides at the $D_{1} \bar{D}$ threshold; there are two $D_{1}$ states, a narrow one with a mass of $2420 \mathrm{MeV}$ and a width of $25 \mathrm{MeV}$, and a wide one with a mass of $2430 \mathrm{MeV}$ and a width of about $400 \mathrm{MeV}$, both decaying into $D^{*} \pi$ [15]. In the former case one has $\lambda \approx 0.05$, while in the latter case $\lambda \approx 0.8$. 


\section{MODIFICATION OF THE WEINBERG FORMULAE}

The Weinberg formalism establishes a relation between low-energy observables, such as the effective-range parameters [10] or Flatté parameters [9], and the nature of a resonance. In particular, it allows one to quantify the admixture of a genuine compact $X_{0}$ component and a molecular component in the physical state $X$ in a model-independent way. The formalism was designed to deal with two-body final states, and in this chapter we address the question as to how the formulae are modified by the presence of an unstable constituent.

The central quantity is the spectral density which, in the near-threshold region, takes the form [see Eqs. (41) and (46)]

$$
W(E)=\frac{1}{2 \pi i}\left[\frac{1}{E-E_{X}+\frac{1}{2} g_{a b} \kappa_{\mathrm{eff}}-\frac{i}{2} g_{a b} k_{\mathrm{eff}}}-\frac{1}{E-E_{X}+\frac{1}{2} g_{a b} \kappa_{\mathrm{eff}}+\frac{i}{2} g_{a b} k_{\mathrm{eff}}}\right],
$$

which is nonzero above the lowest relevant threshold (in our case for $E>0$ ). In the absence of three-body bound states it is normalised to unity,

$$
\int_{0}^{\infty} W(E) d E=1
$$

In the vicinity of the threshold at $E=E_{R}$ the integral

$$
\int_{E_{R}-\delta}^{E_{R}+\delta} W(E) d E
$$

measures the admixture of the bare state for the near-threshold resonance. Indeed, if this integral appears of order unity, then one deals with a genuine quarkonium while a small integral value indicates a dynamical origin of the resonance. Therefore, fitting the production rate (42) with the formula (57), one can estimate the admixture of the bare $X_{0}$ state in the wave function of the $X$-resonance in the near-threshold region defined by the parameter $\delta$ [9]. Most naturally, the scale for $\delta$ is set by the visible width of the resonance, while other scales, like the distance from the next threshold $\left[D^{+} \bar{D}^{*-}\right.$ in the case of the $X(3872)$ particle] could also be relevant.

In the narrow-width limit (56) one can proceed further. Strictly speaking, with the finite width of particle $b$ taken into account (and in the absence of three-body or two-body bound states), the physical space is spanned by the genuine three-body continuum, and the notion of the $a b$ on-shell scattering amplitude ceases to exist. Nevertheless, for small enough energies and in the limit (56), the $t$-matrix element $t_{22}$ takes the form

$$
t_{22}(E)=\frac{1}{8 \pi^{2} \mu_{p}} \frac{g_{a b}}{E-E_{X}+\frac{i}{2} g_{a b} k_{\mathrm{eff}}+\frac{1}{2} g_{a b} \kappa_{\mathrm{eff}}},
$$


which can be viewed, for $\Gamma_{R} \rightarrow 0$, as a Flatté-type representation for the $t$-matrix in the $a b$ channel.

With expression (60) one can study the structure of singularities in the complex plane. If one of the particles is unstable, the Riemann surface does not show a two-body but a three-body cut (if the unstable particle predominantly decays via a two-particle decay, as is assumed throughout this paper). As shown above, in the narrow-width limit, one can replace the expressions for the $k_{\text {eff }}(E)$ and $\kappa_{\text {eff }}(E)$ by the simple ones, as given by Eqs. (6) and (17) with a constant width. Then the $t$-matrix poles are

$$
E_{1,2}=E_{X}+\frac{1}{2} g_{a b} \kappa_{X}-\frac{1}{4} g_{a b}^{2} \mu_{p} \pm \sqrt{\frac{1}{16} g_{a b}^{4} \mu_{p}^{2}-\frac{1}{2} g_{a b}^{2} \mu_{p}\left(E_{X}-E_{R}+\frac{1}{2} g_{a b} \kappa_{X}\right)-\frac{i}{4} g_{a b}^{2} \mu_{p} \Gamma_{R},}
$$

with $\kappa_{X}=\kappa_{\text {eff }}^{(0)}\left(E_{X}\right)$. In the limit of a stable particle $b, \Gamma_{R}=0$, and for $E_{X}<E_{R}$, there is a bound state in the $a b$ system, which corresponds to the upper sign above,

$$
E_{1} \rightarrow E_{B}=E_{X}
$$

and it is straightforward to verify that this pole is situated at the first sheet of the bona fide Riemann surface of the genuine two-body case. For $\Gamma_{R}>0$ the system couples to the sheet with $a, b$, and $c$ on-shell, and what used to be the physical sheet for stable constituents, is now the unphysical one. However, the pole $E_{1}$ remains to be the one most relevant for this discussion. In its vicinity, $t_{22}$ takes the form

$$
t_{22}(E) \rightarrow \frac{g_{\mathrm{eff}}^{2}}{E-E_{B}},
$$

where

$$
\begin{aligned}
g_{\mathrm{eff}}^{2} & =\frac{g_{a b}}{8 \pi^{2} \mu_{p}}\left[1-\frac{1}{2} g_{a b} \frac{\partial}{\partial E}\left(\kappa_{\mathrm{eff}}+i k_{\mathrm{eff}}\right)_{\mid E=E_{B}}\right]^{-1} \\
& =\frac{g_{a b}}{8 \pi^{2} \mu_{p}} \frac{\sqrt{-2 \mu_{p}\left(E_{B}-E_{R}+\frac{i}{2} \Gamma_{R}\right)}}{\sqrt{-2 \mu_{p}\left(E_{B}-E_{R}+\frac{i}{2} \Gamma_{R}\right)}+\frac{1}{2} \mu_{p} g_{a b}} .
\end{aligned}
$$

In the limit (56) and for $E<E_{R}$ one has $k_{\text {eff }} \rightarrow 0$. Then

$$
W(E) \rightarrow \frac{\delta\left(E-E_{B}\right)}{1-\frac{1}{2} g_{a b} \frac{\partial}{\partial E} \sqrt{-2 \mu_{p}\left(E-E_{R}\right)_{\mid E=E_{B}}}}=\mathcal{Z} \delta\left(E-E_{B}\right) .
$$

The factor $\mathcal{Z}$ on the right-hand side of Eq. (65) is nothing but the probability to find the bare state in the wave function of the bound state. 
Equation (65) allows one to express the coupling $g_{a b}$ in terms of $\mathcal{Z}$ and $E_{B}$. Then the vertex $g_{\text {eff }}$ takes the famous Weinberg form [10]

$$
g_{\text {eff }}^{2} \rightarrow \frac{\sqrt{-2 \mu_{p}\left(E_{B}-E_{R}\right)}}{4 \pi^{2} \mu_{p}^{2}}(1-\mathcal{Z})
$$

As it was argued previously [10], the given expression is correct only up to terms of order $\mathcal{O}\left(\sqrt{2 \mu_{p}\left(E_{R}-E_{B}\right)} / \beta\right)$, where $\beta$ denotes the range of forces. Our analysis reveals that in case of unstable constituents there are, in addition to the finite-range corrections, also corrections of order $\lambda$. However, for $\lambda \ll 1$ the Weinberg analysis should still be applicable.

\section{LINESHAPES}

We are now in a position to discuss the lineshapes of composite particles with unstable constituents. For the plots shown below we consider the $X(3872)$, as a paradigmatic example, which allows us to quantify also the effect of the inelastic channels in line with Refs. [3, 4].

We start with the expression for the spectral density, similar to the one given by Eq. (57),

$$
W(E)=\frac{1}{2 \pi i}\left[\frac{1}{E-E_{X}+G_{X}^{*}(E)}-\frac{1}{E-E_{X}+G_{X}(E)}\right],
$$

with

$$
G_{X}(E)=\frac{1}{2} g_{a b} \kappa_{\mathrm{eff}}+\frac{i}{2}\left(g_{a b} k_{\mathrm{eff}}+\Gamma_{0}\right) .
$$

In this expression we introduced, in addition to what we had before, the quantity $\Gamma_{0}$ to mimic inelastic channels (for example, the channel $\pi \pi J / \psi$ in case of the $X(3872)$ ).

Then the differential branching fractions are given by (as mentioned before, we absorb all details of the $X$ production into the constant $\mathcal{B}$ ):

$$
\frac{d \operatorname{Br}(a[c d])}{d E}=\mathcal{B} \frac{1}{2 \pi} \frac{g_{a b} k_{\mathrm{eff}}(E)}{\left(E-E_{X}+\frac{g_{a b}}{2} \kappa_{\mathrm{eff}}(E)\right)^{2}+\frac{1}{4}\left(\Gamma_{0}+g_{a b} k_{\mathrm{eff}}(E)\right)^{2}},
$$

for the $a[c d]$ channel $(D D \pi$ channel for the $X(3872))$, and

$$
\frac{d \mathrm{Br}(\mathrm{inel})}{d E}=\mathcal{B} \frac{1}{2 \pi} \frac{\Gamma_{0}}{\left(E-E_{X}+\frac{g_{a b}}{2} \kappa_{\mathrm{eff}}(E)\right)^{2}+\frac{1}{4}\left(\Gamma_{0}+g_{a b} k_{\mathrm{eff}}(E)\right)^{2}},
$$

for the inelastic channel.

To illustrate the effect of the finite width of one of the constituents we investigate the above-mentioned differential branching ratios for both the $S$ - and $P$-wave case and for both 

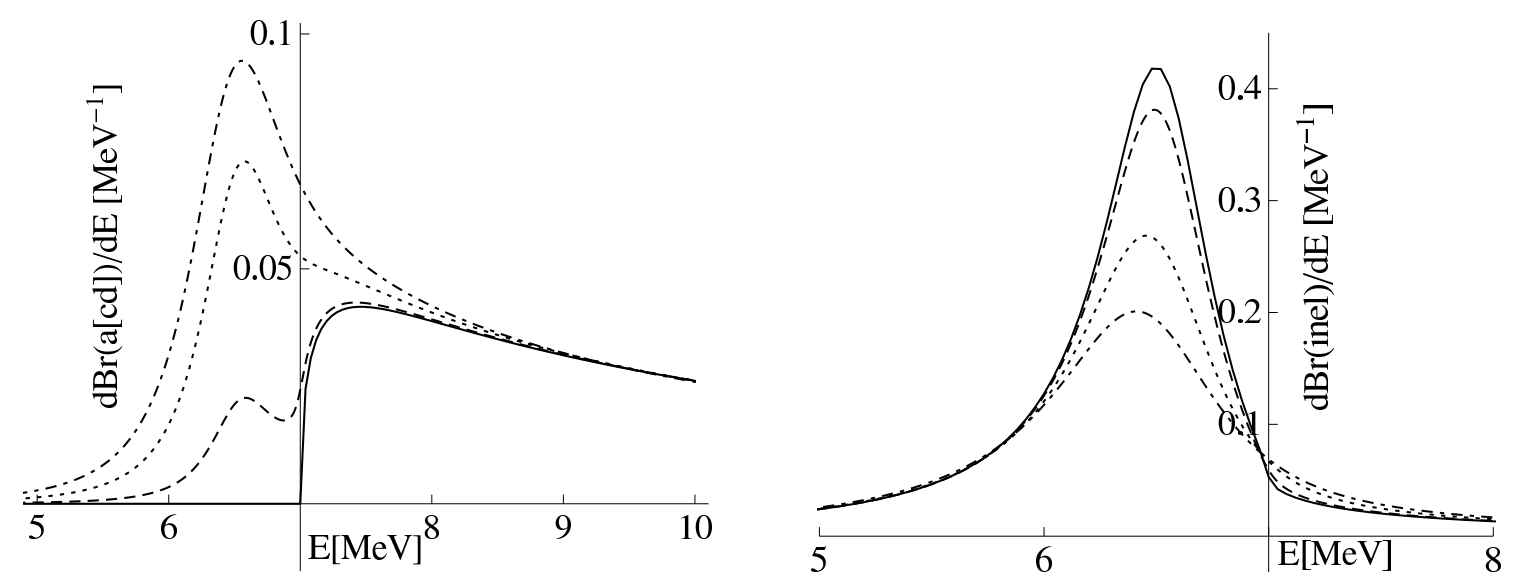

FIG. 6: The $a[c d]$ production rate $[D D \pi$ rate for the $X(3872)]$ (the first plot) and the inelastic rate $[\pi \pi J / \psi$ for the $X(3872)]$ (the second plot) for the $S$-wave resonance and for the $X$ being a bound state. Here $E_{R}=7 \mathrm{MeV}$ and $\Gamma_{R}=0$ (solid line), $\Gamma_{R}=100 \mathrm{keV}$ (dashed line), $\Gamma_{R}=500$ $\mathrm{keV}$ (dotted line), and $\Gamma_{R}=1 \mathrm{MeV}$ (dash-dotted line). Notice that, since we are interested in the lineshapes, the above rates are plotted for $\mathcal{B}=1$.
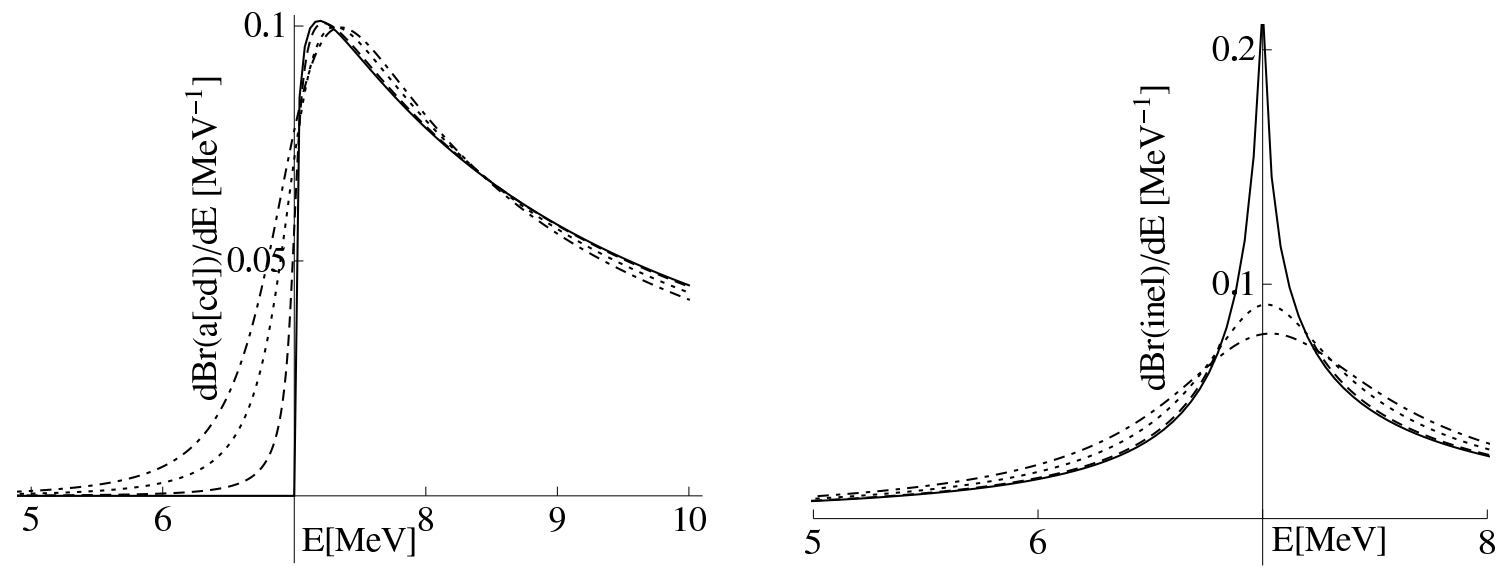

FIG. 7: The same as in Fig. 6, but for the $X$ being a virtual state.

bound and virtual $X$ particles. However, before we proceed, let us remind the reader of the definition of bound and virtual states and show their relation to the parameters introduced above. Consider the case when all inelasticities are switched off. Then $k_{\text {eff }}(E)$ and $\kappa_{\text {eff }}(E)$ are given by the simple formulae (3) and (44). To distinguish between bound- and virtualstate situations it is convenient to consider the poles of the denominator $E-E_{X}+G_{X}(E)$ in the $k$-plane, where $k=\sqrt{2 \mu\left(E-E_{R}\right)}$. For $E_{X}<E_{R}$ these poles are

$$
k_{1}=i \sqrt{2 \mu_{p}\left(E_{R}-E_{X}\right)}, \quad k_{2}=-i \sqrt{2 \mu_{p}\left(E_{R}-E_{X}\right)}-i g_{a b} \mu_{p} .
$$



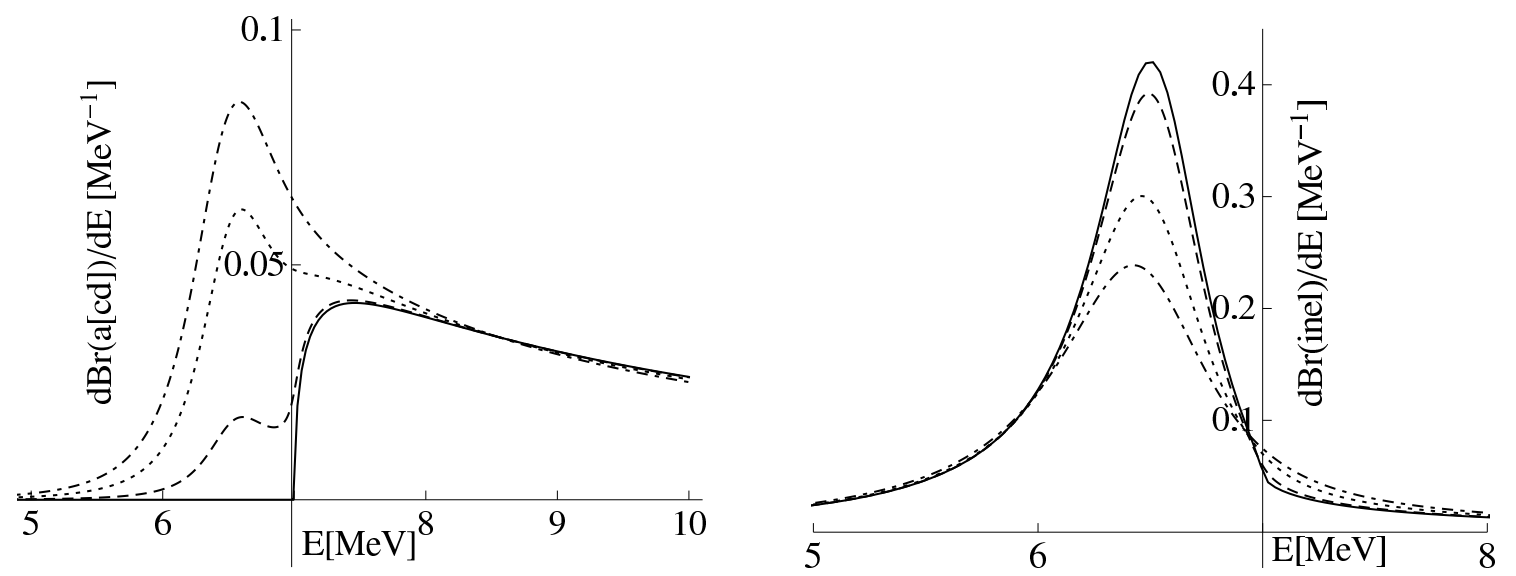

FIG. 8: The same as in Fig. 6, but for the $P$-wave resonance.
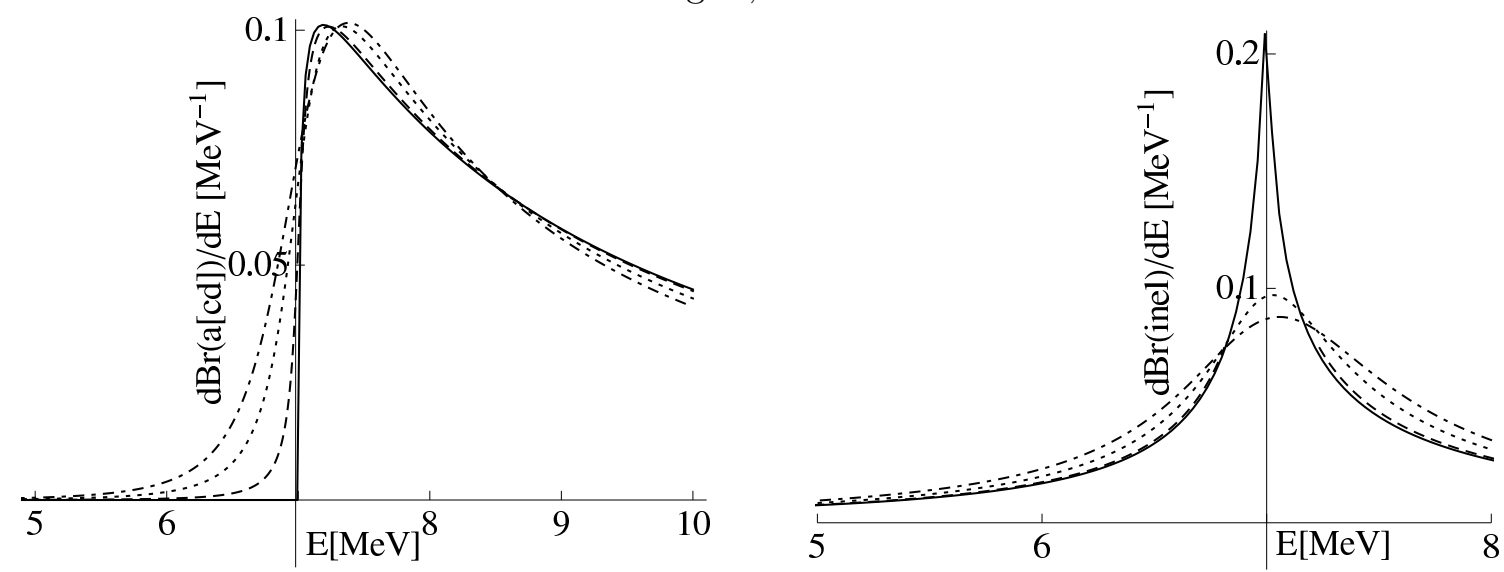

FIG. 9: The same as in Fig. 7, but for the $P$-wave resonance.

The first pole is situated on the first sheet of the genuine two-body Riemann surface, and corresponds to the bound state with the energy $E_{X}$, and the second pole is at the unphysical sheet. The scattering length in the $a b$ channel is positive for this case. On the contrary, for $E_{X}>E_{R}$ the poles are:

$$
k_{1,2}=-i \frac{g_{a b} \mu_{p}}{2} \pm i \sqrt{\frac{g_{a b}^{2} \mu_{p}^{2}}{4}-2 \mu_{p}\left(E_{X}-E_{R}\right)}
$$

Both poles are located on the unphysical sheet, and the scattering length is negative. For $g_{a b}^{2} \mu_{p}>8\left(E_{X}-E_{R}\right)$ the poles are on the imaginary axis, and we deal with a virtual state. For small inelasticities, the scattering length acquires a small imaginary part, so that we distinguish between the bound and the virtual states by the sign of the real part of $a$. Thus we plot in Figs. 66 the differential branching ratios (69) and (70) for both the $S$ - and $P$ wave case and $E_{X}<E_{R}$ (bound state) and $E_{X}>E_{R}$ (virtual state). In particular, for the 
bound-state case we take

$$
E_{R}=7 \mathrm{MeV}, \quad g_{a b}=0.1, \quad E_{X}=E_{R}-0.5 \mathrm{MeV}, \quad \Gamma_{0}=1.5 \mathrm{MeV} .
$$

This set of parameters is similar to the one obtained in Ref. [13] from the fitting Belle data on the $D^{0} \bar{D}^{0} \pi^{0}$ and $\pi^{+} \pi^{-} J / \psi$ decay modes of the $X(3872)$ particle.

As one can see from Figs. 6 and 8, for an increasing width of the constituent, the lineshape in the $a[c d]$ channel starts to develop a peak below the nominal threshold, since the finite width of the unstable constituent allows for an overlap with the subthreshold pole. If the width is not large, the lineshape above threshold is not affected while, with an increase of the width of particle $b$, the structure present below the nominal $a b$ threshold starts to grow very quickly, and it becomes broader. Indeed, already for a width of $1 \mathrm{MeV}$, the structure below the nominal threshold has grown in magnitude and width so much that the whole lineshape is just a single broad bump, however, with a noticeable asymmetry letting it deviate significantly from the standard Breit-Wigner form. The structure for the inelastic channel decreases rapidly with the increase of the width of particle $b$. The mentioned kind of asymmetry, found experimentally in the spectral distribution for the $Y(4660)$ observed in $\psi^{\prime} \pi \pi$ [8], was interpreted in Ref. [16], fully in line with the reasoning presented in this work, as a clear signal for a dominant molecular $f_{0}(980) \psi^{\prime}$ component in the $Y(4660)$. In addition, the same kind of asymmetry was predicted to show up from an analogous $\eta_{c}^{\prime} f_{0}(980)$ bound system in the $\eta_{c}^{\prime} \pi \pi$ invariant mass distribution, yet to be found [17].

For the virtual-state case we take the same values for the $g_{a b}$ and $\Gamma_{0}$ as for the boundstate case - see Eq. (73) - but put $E_{X}=E_{R}+0.5 \mathrm{MeV}$. Then, as seen from Figs. 7 and 9, the effect of the finite width on the $a[c d]$ lineshape is less dramatic since, if the $X$ is a virtual state, no pole develops below the threshold. This is already an unambiguous decisive difference between the bound state and the virtual state. The inelastic rate decreases with the increase of the width of particle $b$, and the cusplike lineshape, present in the zero-width limit, smoothens, so that the shape for the virtual state becomes hardly distinguishable from that for the bound state, however, with a shifted peak.

It follows from the reasoning of Ref. [9] that the height of the hump above the nominal $a b$ threshold contains important information on the nature of the state. We would therefore like to repeat here the argument given in Refs. [6, 13] that it is dangerous to apply a kinematic fit to the data, which moves all relevant signals to above threshold: in this way the signal 
above the nominal threshold would be risen at the cost of the signal below the threshold. Therefore, this procedure might produce data that call for a very different interpretation of the $X$ state than that for the original data set.

\section{INTERFERENCE}

In this chapter we discuss one more effect which plays a role for the lineshapes, namely the interferences in the final states [18]. For example, the $X(3872)$ decays in equal parts via $\bar{D}^{0} D^{0 *} \rightarrow \bar{D}^{0}\left[D^{0} \pi^{0}\right]$ or $D^{0} \bar{D}^{0 *} \rightarrow D^{0}\left[\bar{D}^{0} \pi^{0}\right]$ and, clearly, the final states of both decay chains interfere.

If taken naively, the interference can be taken into account through the modification [see Eq. (37)]

$$
C(\tilde{\boldsymbol{p}}, \tilde{\boldsymbol{q}}) \rightarrow \tilde{C}(\tilde{\boldsymbol{p}}, \tilde{\boldsymbol{q}})=\frac{1}{\sqrt{2}}(C(\tilde{\boldsymbol{p}}, \tilde{\boldsymbol{q}})+C(\overline{\boldsymbol{p}}, \overline{\boldsymbol{q}}))
$$

in Eq. (40), where $\overline{\boldsymbol{p}}$ and $\overline{\boldsymbol{q}}$ are known linear combinations of the momenta $\tilde{\boldsymbol{p}}$ and $\tilde{\boldsymbol{q}}$ :

$$
\overline{\boldsymbol{p}}=-\frac{m_{a}}{m_{c}+m_{d}} \tilde{\boldsymbol{p}}+\tilde{\boldsymbol{q}}, \quad \overline{\boldsymbol{q}}=\frac{m_{a}}{m_{c}+m_{d}} \tilde{\boldsymbol{q}}+\frac{\left(m_{a}+m_{c}+m_{d}\right) m_{d}}{\left(m_{c}+m_{d}\right)^{2}} \tilde{\boldsymbol{p}} .
$$

The phase-space integration measure is invariant under the variable change $\{\tilde{\boldsymbol{p}}, \tilde{\boldsymbol{q}}\} \rightarrow$ $\{\overline{\boldsymbol{p}}, \overline{\boldsymbol{q}}\}$ and the modified Eq. (40) reads

$$
W(M)=\int d^{3} \tilde{p} d^{3} \tilde{q}\left[|C(\tilde{\boldsymbol{p}}, \tilde{\boldsymbol{q}})|^{2}+\operatorname{Re}\left(C(\tilde{\boldsymbol{p}}, \tilde{\boldsymbol{q}}) C^{*}(\overline{\boldsymbol{p}}, \overline{\boldsymbol{q}})\right)\right] \delta\left(M-m_{a}-m_{c}-m_{d}-T_{3}(\tilde{p}, \tilde{q})\right),
$$

where the second term in the square brackets describes the interference. It is clear then that the interference simply produces an extra contribution to be added to $k_{\text {eff }}(E)$, which we denote as $k_{\text {int }}(E)$ and which can be extracted from Eq. (76) in the form

$$
k_{\mathrm{int}}(E)=k_{\mathrm{eff}}(E) \frac{\int d^{3} \tilde{p} d^{3} \tilde{q} \operatorname{Re}\left(C(\tilde{\boldsymbol{p}}, \tilde{\boldsymbol{q}}) C^{*}(\overline{\boldsymbol{p}}, \overline{\boldsymbol{q}})\right) \delta\left(M-m_{a}-m_{c}-m_{d}-T_{3}(\tilde{p}, \tilde{q})\right)}{\int d^{3} \tilde{p} d^{3} \tilde{q}|C(\tilde{\boldsymbol{p}}, \tilde{\boldsymbol{q}})|^{2} \delta\left(M-m_{a}-m_{c}-m_{d}-T_{3}(\tilde{p}, \tilde{q})\right)} .
$$

Therefore, in leading order in the interference, the $a[c d]$ rate can be written in the form of Eq. (69) but with the $k_{\text {eff }}(E)$ in the numerator substituted by the sum $k_{\text {eff }}(E)+k_{\text {int }}(E)$ :

$$
\frac{d \operatorname{Br}(a[c d])}{d E}=\mathcal{B} \frac{1}{2 \pi} \frac{g_{a b}\left(k_{\mathrm{eff}}(E)+k_{\mathrm{int}}(E)\right)}{\left(E-E_{X}+\frac{g_{a b}}{2} \kappa_{\mathrm{eff}}(E)\right)^{2}+\frac{1}{4}\left(\Gamma_{0}+g_{a b} k_{\mathrm{eff}}(E)\right)^{2}} .
$$



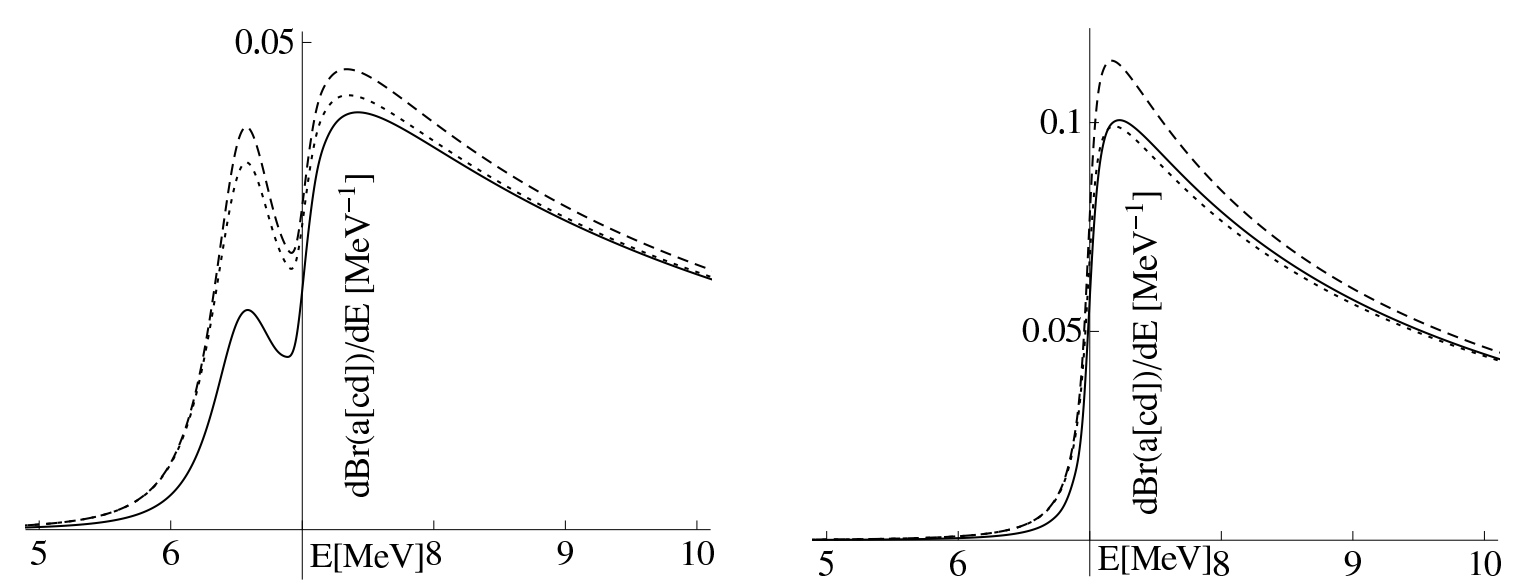

FIG. 10: The $a[c d]$ state production rate for the $S$-wave resonance for bound-state case (the first plot) and for the virtual-state case (the second plot). Solid line: no interference; dashed line: naive interference; and dotted line: improved interference. As before, we set $\mathcal{B}=1$.

Clearly, taking the interference into account in the numerator only while neglecting it in the denominator (higher orders in the interference) leads to a violation of unitarity, which can be cured by solving the full coupled-channel problem with the effects of dynamical building blocks of the constituents taken into account from the very beginning. However, bearing in mind a very moderate effect of the interference over the lineshapes, we resort to a much simpler approach which amounts to taking $k_{\text {int }}(E)$ into account both in the numerator and in the denominator simultaneously:

$$
\frac{d \operatorname{Br}(a[c d])}{d E}=\mathcal{B} \frac{1}{2 \pi} \frac{g_{a b}\left(k_{\mathrm{eff}}(E)+k_{\mathrm{int}}(E)\right)}{\left(E-E_{X}+\frac{g_{a b}}{2} \kappa_{\mathrm{eff}}(E)\right)^{2}+\frac{1}{4}\left(\Gamma_{0}+g_{a b}\left(k_{\mathrm{eff}}(E)+k_{\mathrm{int}}(E)\right)\right)^{2}} .
$$

We call this an improved interference.

In Fig. 10 we plot the rate $d \operatorname{Br}(a[c d]) / d E$ for an $S$-wave resonance and for the following three cases: (i) no interference [as given by Eq. [69)], (ii) naive interference [as given by Eq. (78)], and (iii) improved interference [as given by Eq. (79)]. For definiteness, we fix all parameters compatible with the $X(3872)$ charmonium, so that the parameters used are

$$
E_{R}=7 \mathrm{MeV}, \quad g_{a b}=0.1, \quad E_{X}=E_{R} \mp 0.5 \mathrm{MeV}, \quad \Gamma_{0}=1.5 \mathrm{MeV}, \quad \Gamma_{R}=100 \mathrm{keV}
$$

where upper (lower) sign in $E_{X}$ corresponds to bound(virtual)-state case. Finally, we set $m_{a}=m_{c}=m_{0}$ and $m_{d}=m_{\pi}$, with $m_{0}$ and $m_{\pi}$ being the masses of the $D^{0}$ and $\pi^{0}$ mesons, respectively. 
The corresponding plots for the $P$-wave exhibit exactly the same behaviour as for the $S$-wave and are omitted here.

As one could anticipate, the net effect of the interference is an increase of the rate in the region of the bound-state peak, while the above-threshold rate remains practically the same.

A proper account for the interference obviously requires a more rigorous treatment of the coupled-channel problem. Indeed, in the case of the $X(3872)$, a potentially important piece of interaction is omitted in the Hamiltonian (16), which is responsible for the $D^{0} \bar{D}^{0 *}$ $\bar{D}^{0} D^{0 *}$ transition, namely the pion exchange. This interaction is often considered as the main binding mechanism in the $D^{0} \bar{D}^{0 *}$ system [19-21], and the $X$ is generated dynamically, similarly to the deuteron. It was noticed, however, that, as the nominal threshold for $D^{0} \bar{D}^{0 *}$ is only about $7 \mathrm{MeV}$ larger than the three-body $D^{0} \bar{D}^{0} \pi^{0}$ threshold, the pion may go on-shell and, as a result, such a binding may not be strong enough (see, for example, Ref. [22]). It was argued in Ref. [23] that the effect of the $\pi^{0}$ exchange for the $D^{0} \bar{D}^{0 *}$ system can be treated perturbatively, in contrast to the two-nucleon system. If it is indeed the case, the expression (178) for the $D^{0} \bar{D}^{0} \pi^{0}$ rate should be appropriate. This statement is further supported by the observation that the rates described with the "naive" and the "improved" prescriptions for the interference do not differ a lot.

\section{SUMMARY}

In this paper we investigated the effect of the finite width of a constituent of a composite state on its lineshape. In a coupled-channel formalism we derived exact formulae for such lineshapes and compared them to simple prescriptions found in the literature. We found that such simple prescriptions work only for extremely narrow constituents located far away from the three-body threshold characterised by $\lambda=\Gamma_{R} /\left(2 E_{R}\right) \ll 1$, where $\Gamma_{R}$ denotes the width of the constituent and $E_{R}$ its resonance energy, measured with respect to the three-body threshold. In this regime the resonance width can safely be treated as energy independent. The famous charmonium state $X(3872)$ gives an example of such a safe situation with $\lambda \ll 1$ in view of an extremely small width of the $D^{*}$ meson. However, for an increasing width of the constituent (and correspondingly an increasing value of $\lambda$ ), the exact formulae start to deviate significantly from the simplified ansatz. The situation cannot be improved by using an energy-dependent width for the constituent instead of a constant width - the three-body 
threshold behaviour gets spoilt in this case, while the near-two-body threshold behaviour is not improved. We argue therefore that the exact formulae have to be employed in such a situation.

The effect of the finite width of a constituent on the lineshape in the elastic channel of the composite state $X$ is quite moderate, if the $X$ is a virtual state. However, for the $X$ being a bound state, a nonnegligible bound-state peak starts to develop below the nominal threshold for an increasing constituent width, as already stressed in Ref. [3]. For sufficiently large values of the width, the bound-state peak becomes broad enough to distort the lineshape both below and above the threshold, so that the resulting lineshape takes the form of a broad hump that one can hardly distinguish in shape from the virtual-state situations. However, the peak position appears to be shifted, since for the virtual state the distribution is peaked above the nominal two-body threshold while for the bound state it is peaked below. Clearly, to distinguish the two experimentally calls for a quite high resolution.

The inelastic production rate is less affected by the finite-width effects. However, only for extremely small values of the width one is able to distinguish between the threshold cusp scenario, in case of the virtual state, and a smooth below-threshold peak, in case of the bound state. For an increasing width, a smeared broad structure is developed in both cases. This makes it difficult to distinguish between the two scenarios, although the peaks of the two distributions appear to be shifted also in this case. It is important to stress that the distributions turn out to be very asymmetric — inconsistent with a Breit-Wigner shape - in both cases. This asymmetry can be taken as a strong signal towards a composite two-hadron structure in distinction to a genuine, compact quark state.

As a related problem, we studied the interference in the final state. For the sake of definiteness, we focused on the case of the $X(3872)$ charmonium and fixed all free parameters accordingly. The conclusion we find is that, while the effect of the interference on the elastic lineshape is indeed sizable for the bound state and below the nominal threshold, in line with the findings reported in the literature, this effect is negligible for the bound state above the threshold as well as for the virtual state both below and above the threshold. However, for an increasing width one is to include the effects of interference. Furthermore, the naive inclusion of the interference meets severe problems with unitarity, so that, while for intermediate values of the width, an improved interference suggested in this paper should be used, for a width large enough one is finally forced to solve the full coupled-channel problem 
with the effects of dynamical building blocks of the constituents taken into account from the very beginning.

We treat our findings as an important step in building a universal lineshape parameterisation scheme suitable for fitting experimental data for various near-threshold resonances and thus we believe that our insights will prove useful for the analysis of future experiments.

\section{Acknowledgments}

We would like to thank Eric Braaten, Feng-Kun Guo, and Bruce Yabsley for valuable comments. This work was supported in parts by funds from the Helmholtz Association (grants VH-NG-222, VH-VI-231), by the DFG (grants SFB/TR 16 and 436 RUS 113/991/01), by the EU HadronPhysics2 project, by the RFFI (grants RFFI-09-02-91342-NNIOa and RFFI-09-02-00629a), and by the Presidential programme for support of the leading scientific schools (grant NSh-4961.2008.2). Work of Yu. K. and A. N. was supported by the State Corporation of Russian Federation "Rosatom." A. N. would also like to acknowledge the support of the grants PTDC/FIS/70843/2006-Fisica, and of the nonprofit Dynasty foundation and ICFPM.

[1] E. J. Eichten, K. Lane, and Ch. Quigg, Phys. Rev. D 73, 014014 (2006).

[2] S. L. Olsen, Nucl. Phys. A827, 53C (2009).

[3] E. Braaten and M. Lu, Phys. Rev. D 76, 094028 (2007).

[4] C. Hanhart, Yu. S. Kalashnikova, A. E. Kudryavtsev, and A. V. Nefediev, Phys. Rev. D 76, 034007 (2007).

[5] M. Nauenberg and A. Pais, Phys. Rev. 126, 360 (1962).

[6] E. Braaten and J. Stapleton, arXiv:0907.3167[hep-ph].

[7] B. Aubert et al. [BABAR Collaboration], Phys. Rev. Lett. 95, 142001, (2005).

[8] X. L. Wang et al. [Belle Collaboration], Phys. Rev. Lett. 99, 142002, (2007).

[9] V. Baru, J. Haidenbauer, C. Hanhart, Yu. Kalashnikova, and A. Kudryavtsev, Phys. Lett. B 586, $53(2004)$. 
[10] S. Weinberg, Phys. Rev. 130, 776 (1963); Phys. Rev. 131, 440 (1963); Phys. Rev. 137, B672 (1965).

[11] V. Baru, C. Hanhart, Yu. S. Kalashnikova, A. E. Kudryavtsev, and A. V. Nefediev, Eur. Phys. J. A 44, 93 (2010).

[12] M. Doring, C. Hanhart, F. Huang, S. Krewald and U.-G. Meißner, Nucl. Phys. A 829, 170, (2009).

[13] Yu. S. Kalashnikova and A. V. Nefediev, Phys. Rev. D 80, 074004 (2009).

[14] C. Hanhart, J. R. Pelaez, and G. Rios, Phys. Rev. Lett. 100, 152001 (2008).

[15] C. Amsler et al. (Particle Data Group), Phys. Lett. B 667, 1 (2008).

[16] F. K. Guo, C. Hanhart and U.-G. Meißner, Phys. Lett. B 665, 26 (2008).

[17] F. K. Guo, C. Hanhart, and Ulf-G. Meißner, Phys. Rev. Lett. 102, 242004, (2009).

[18] M. B. Voloshin, Phys. Lett. B 579, 316 (2004).

[19] M. B. Voloshin and L. B. Okun, Pisma Zh. Eksp. Teor. Fiz. 23, 369 (1976) (JETP Lett. 23, $333(1976))$.

[20] N. A. Tornqvist, Phys. Rev. Lett. 67, 556 (1991); Phys. Lett. B 590, 209 (2004).

[21] E. S. Swanson, Phys. Lett. B 588, 189 (2004).

[22] M. Suzuki, Phys. Rev. D 72, 114013 (2005).

[23] S. Fleming, M. Kusunoki, T. Mehen, and U. van Kolck, Phys. Rev. D 76, 034006 (2007). 